\title{
STABILITY OF STATIONARY POINTS AND COHOMOLOGY OF GROUPS
}

\author{
MORRIS W. HIRSCH ${ }^{1}$
}

\begin{abstract}
A stationary point of a group action is called stable if nearby actions have nearby stationary points. Sufficient conditions for stability are found for certain linear actions. In special cases necessary and sufficient conditions are determined.
\end{abstract}

Introduction. A stationary point of a group action is called stable if all nearby actions have nearby stationary points (precise definitions are given below). In this paper necessary conditions for stability are found for certain linear actions. In very special cases necessary and sufficient conditions are obtained.

Let $E$ be a finite-dimensional real vector space on which the topological group $G$ acts linearly. Let $H^{i}(G, E)$ denote the cohomology of the resulting $G$-module, based on continuous cochains (see Stasheff [9] for a survey). The set of stationary points in $H^{0}(G, E)$, also denoted by $E^{G}$.

Our results flow from the observation that if the origin $0 \in E$ is a stable stationary point then $H^{1}(G, E)=0$, proved below as Theorem 1.1. The vanishing of $H^{1}$ is equivalent to stability of the origin under translational deformations of the action.

THeOREM A. If the origin is stable then the largest unipotent submodule is $E^{G}$.

THEOREM B. If the origin is stable and there is a nonzero continuous homomorphism $G \rightarrow \mathbf{R}$, then $E^{G}=0$.

The homomorphism requirement cannot be dropped from Theorem B, for if $G$ is compact every stationary point is stable [7].

THeOREM C. Let $G$ be a connected, noncompact, nilpotent Lie group. Then the following are equivalent.

(a) The origin is stable.

(b) $E^{G}=0$.

(c) $I-g$ is invertible for some $g \in G$.

That (b) implies (a) follows from a theorem of Dennis Stowe [10]; his result also shows that if $G$ is not connected then (a) need not imply (c). There is, however, the following result.

\footnotetext{
Received by the editors November 1, 1978 and, in revised form, March 15, 1979.

AMS (MOS) subject classifications (1970). Primary 18H10, 57E20, 57E30, 58F10; Secondary 22E25, $55 \mathrm{C20}$.

${ }^{1}$ Supported in part by NSF Grant MCS 74-93423.A02. 
THEOREM D. Let $G$ be a discrete, finitely-generated, nilpotent group with $\operatorname{Hom}(G, \mathbf{R}) \neq 0$. Then the origin is stable if and only if $E^{G}=0$.

Theorems $\mathrm{C}$ and $\mathrm{D}$ are proved in $\$ 3$.

1. Stability implies $H^{1}(G, E)=0$. The group Diff $E$ of $C^{1}$ diffeomorphisms of $E$ is given the weak $C^{1}$ topology [4]. An action of $G$ on $E$ is a continuous homomorphism $\alpha: G \rightarrow \operatorname{Diff} E$. The set $\mathcal{Q}(G, E)$ of all actions of $G$ on $E$ is given the compact open topology inherited from the space of all continuous maps of $G$ into Diff $E$.

We assume the action $\alpha$ is fixed in any discussion; for $g \in G, x \in E$ we may write $g x$ in place of $\alpha(g) x$.

Let $p \in E$ be a stationary point for the action $\alpha$, that is, $\alpha(g) x=x$ for all $g$. We call $p$ stable if for every neighborhood $U \subset E$ of $p$ there exists a neighborhood $\Re \subset \mathbb{Q}(G, E)$ of $\alpha$ such that every action in $\Re$ has a stationary point in $U$. Various sufficient conditions for stability are developed in Hirsch [6], [7].

Fix a linear action $\alpha: G \rightarrow G L(E)$; this makes $E$ into a $G$-module. A crossed homomorphism, or cocycle, $u: G \rightarrow E$ is a continuous map such that

$$
u(g h)=u(g)+g u(h), \quad(g, h \in G) .
$$

As is easily verified this is exactly the condition that the following map $\alpha_{u}$ from $G$ into the group of affine automorphisms be a homomorphism:

$$
\alpha_{u}: G \rightarrow \operatorname{Aff}(E), \quad \alpha_{u}(g): x \mapsto g x+u(g), \quad(x \in E, g \in G) .
$$

The crossed homomorphism $u$ is called principal if there exists $y \in E$ such that $u(g)=(I-g) y$ for all $g$. This is precisely the condition that $y$ be a stationary point of the action $\alpha_{u}$.

The vector space $H^{1}(G, E)$ is defined as the factor space of crossed homomorphisms modulo principal ones.

The only tools needed from the cohomology of groups are the inflation-restriction sequence and the exact sequence associated to coefficient homomorphisms, in dimensions $\mathbf{0}$ and 1 only. The continuous theory is almost the same as the discrete one as presented, for example, in Atiyah-Wall [1].

1.1. THEOREM. If the origin is a stable stationary point for a linear action $\alpha$ then $H^{1}(G, E)=0$.

Proof. Let $u: G \rightarrow E$ be a crossed homomorphism. For every $t \in \mathbf{R}$ there is defined the affine action $\alpha_{t u}$ of $G$ on $E$ (see (1) above). As $t \rightarrow 0, \alpha_{t u} \rightarrow \alpha$ in $\mathbb{Q}(G, E)$. It follows from stability that for $t$ near $0, \alpha_{t u}$ has a stationary point. Hence $t u$ represents 0 in $H^{1}(G, E)$ for some $t \neq 0$; this implies the theorem. Q.E.D.

There is nothing special about the origin in Theorem 1.1; the same result holds for any stationary point $p$ of a linear action. This becomes obvious when one conjugates the action with translation by $p$. 
2. Proof of Theorems $A$ and B. Let $E$ be a real, finite-dimensional $G$-module determined by $\alpha: G \rightarrow \mathrm{GL}(E)$. Let $E_{U} \subset E$ be the largest linear subspace on which each element of $G$ is unipotent. $\operatorname{Hom}(G, \mathbf{R})$ denotes the vector space of continuous homomorphisms from $G$ to $\mathbf{R}$.

In view of Theorem 1.1, Theorems $A$ and $B$ are implied respectively by

2.1. Theorem. If $H^{1}(G, E)=0$ then $E_{U}=E^{G}$.

2.2. TheOREM. If $\operatorname{Hom}(G, \mathbf{R}) \neq 0$ and $H^{1}(G, E)=0$, then $H^{0}(G, E)=0$.

Proof of Theorem 2.1. It follows from the definition of $E_{U}$ that $H^{0}\left(G, E / E_{U}\right)$ $=0$. Therefore the short exact sequence of $G$-modules $E_{U} \rightarrow E \rightarrow E / E_{U}$ yields an exact cohomology sequence containing $0 \rightarrow H^{1}\left(G, E_{U}\right) \rightarrow H^{1}(G, E)$. The hypothesis of Theorem 2.1 thus implies $H^{1}\left(G, E_{U}\right)=0$. Therefore Theorem 2.1 is a consequence of the following result by taking $F=E_{U}$.

2.3. TheOREM. Let $F$ be a real finite-dimensional vector space on which $G$ has a nontrivial unipotent linear action. Then $H^{1}(G, F) \neq 0$.

Proof of TheORem 2.3. The proof proceeds by induction on $n=\operatorname{dim} F$, the cases $n=0,1$ being vacuous. Suppose $n>1$. There is a basis of $F$ putting the representation of $G$ in upper-triangular unipotent form. We fix such a basis, thus identifying $F$ with $\mathbf{R}^{n}$. The element $g$ of $G$ acts by the matrix $A(g)=\left[A_{i j}(g)\right]$, with $A_{i j}(g)=0$ if $i>j$ and $A_{i i}(g)=1$ for $i, j=1, \ldots, n$.

Suppose $n=2$. In this case a nonprincipal crossed homomorphism $v: G \rightarrow \mathbf{R}^{2}$ is defined by the formula

$$
v(g)=\left(A_{12}(g)^{2} / 2, A_{12}(g)\right)
$$

as is easily verified.

Assume now that $n>2$ and that Theorem 2.3 is true for vector spaces of lower dimension.

Let $K \subset G$ be the kernel of the representation $A: G \rightarrow \mathrm{GL}(n, \mathbf{R})$. The stationary set of $K$ is all of $\mathbf{R}^{n}=F$. From the exact inflation-restriction sequence

$$
0 \rightarrow H^{1}\left(G / K, F^{K}\right) \rightarrow H^{1}(G, F)
$$

we see that it suffices to prove that $H^{1}(G / K, F) \neq 0$. Since $G / K$ acts nontrivially and faithfully on $F$, from now on we may assume that $G$ acts faithfully.

Let $J \subset G$ be the kernel of the induced representation of $G$ on the subspace $\mathbf{R}^{n-1} \subset \mathbf{R}^{n}$ spanned by the first $n-1$ basis vectors. Since $J$ acts faithfully on $\mathbf{R}^{n}$ it follows that the stationary set of $J$ is $\mathbf{R}^{n-1}$.

Suppose $J \neq G$. Then $G / J$ acts nontrivially on $\mathbf{R}^{n-1}$ and the induction hypothesis implies $H^{1}\left(G / J, \mathbf{R}^{n-1}\right) \neq 0$. The exact inflation-restriction sequence

$$
0 \rightarrow H^{1}\left(G / J, \mathbf{R}^{n-1}\right) \rightarrow H^{1}\left(G, \mathbf{R}^{n}\right)
$$

shows that Theorem 2.3 holds in this case.

Suppose $J=G$, so that $G$ acts trivially on $\mathbf{R}^{n-1}$. The exact cohomology sequence corresponding to $\mathbf{R}^{n-1} \rightarrow \mathbf{R}^{n} \rightarrow \mathbf{R}^{n} / \mathbf{R}^{n-1}$ contains the exact sequence

$$
H^{0}\left(G, \mathbf{R}^{n} / \mathbf{R}^{n-1}\right) \rightarrow H^{1}\left(G, \mathbf{R}^{n-1}\right) \rightarrow H^{1}\left(G, \mathbf{R}^{n}\right) .
$$


The first term is isomorphic to $\mathbf{R}$ and the middle term to $\operatorname{Hom}\left(G, \mathbf{R}^{n-1}\right)$. This latter group is nontrivial: it is easily checked that the last column of the matrix $A(g)-I$ provides a nonzero homomorphism from $G$ to $\mathbf{R}^{n}$. This shows that $\operatorname{Hom}(G, \mathbf{R}) \neq 0$, which implies that the middle term of the exact sequence has dimension at least $n-1$. Since the first term has dimension 1 and we are assuming $n>2$, it follows that $\operatorname{dim} H^{1}\left(G, \mathbf{R}^{n}\right)>1$. The proofs of Theorems 2.3 and 2.1 are complete.

REMARK. The proof of Theorem 2.3 works under more general hypotheses. For example, $F$ can be a finite dimensional vector space over any field of characteristic $\neq 2$, the cohomology being the usual one (without continuity assumptions). In this situation $G$ might be an algebraic group over the field and the action might be morphic. The same proof then shows that $H_{\text {alg }}^{1}(G, F) \neq 0$, meaning cohomology based on algebraic cocycles.

Proof of Theorem 2.2. As was shown in the proof of Theorem 2.1, if $H^{1}(G, E)$ $=0$ then $H^{1}\left(G, E_{U}\right)=0$. By Theorem 2.1 this makes $H^{1}\left(G, E^{G}\right)=0$. If $\operatorname{Hom}(G, \mathbf{R}) \neq 0$, this implies $E^{G}=0$, proving Theorem 2.1 .

3. Nilpotent groups. In this section $G$ always denotes a nilpotent group acting linearly on a real finite-dimensional vector space $E$.

3.1. Lemma. Suppose $H^{0}(G, E)=0$. Then $H^{i}\left(G, E / E^{\prime}\right)=0$ and $H^{i}\left(G, E^{\prime}\right)=0$ for $i=0,1$, for every submodule $E^{\prime} \subset E$.

Proof. From the exact sequence

$$
0 \rightarrow H^{0}\left(G, E^{\prime}\right) \rightarrow H^{0}(G, E) \rightarrow H^{0}\left(G, E / E^{\prime}\right) \rightarrow H^{1}\left(G, E^{\prime}\right)
$$

we get $H^{0}\left(G, E^{\prime}\right)=0$. By Theorem D of Hirsch [5], this implies $H^{1}\left(G, E^{\prime}\right)=0$. (This uses nilpotence of $G$.) Therefore $H^{0}\left(G, E / E^{\prime}\right)=0$ by exactness, and $H^{1}\left(G, E / E^{\prime}\right)=0$ by [5, Theorem D]. Q.E.D.

It is not hard to prove that for nilpotent $G, H^{0}(G, E)=0$ if and only if $H_{0}(G, E)=0$; compare Fried, Goldman and Hirsch [3]. For nilpotent discrete $G$, Dwyer [2] shows that $H_{0}(G, E)=0$ implies $H_{i}(G, E)=0$ for all $i>0$, while Hirsch [5] shows that $H^{0}(G, E)=0$ implies $H^{i}(G, E)=0$ for all $i>0$.

The following matrix interpretation of Lemma 3.1 is useful. Extend the action of $G$ on $E$ to a $C$-linear action of $G$ on the complexification $C \otimes E$.

3.2. LeMMA. Suppose $\mathrm{C} \otimes E$ has a basis representing the action of $G$ by upper-triangular matrices $\left[A_{i j}(g)\right]$. If $H^{0}(G, E)=0$, then for every $i$ there is a $g \in G$ with $A_{i i}(g) \neq 1$.

Proof. Let the basis be $e_{1}, \ldots, e_{n}$ and let $F_{k} \subset \mathbf{C} \otimes E$ be the $\mathbf{C}$-linear subspace spanned by $e_{1}, \ldots, e_{k}$; put $F_{0}=0$. As a real vector space $\mathbf{C} \otimes E=E \oplus E$. Clearly $H^{0}(G, E \oplus E)=0$. It follows from Lemma 3.1, applied to the $G$-module $\mathrm{C} \otimes E$ and submodule $F_{k}$, that $H^{0}\left(G,(\mathrm{C} \otimes E) / F_{k}\right)=0, k=0, \ldots, n-1$. Now the action of $g \in G$ on $(C \otimes E) / F_{k}$ is by the matrix $\left[A_{i j}(g)\right], i, j=k+1, \ldots, n$, when the images $f_{k+1}, \ldots, f_{n}$ of $e_{k+1}, \ldots, e_{n}$ are chosen as a basis. Because these are upper-triangular, $g\left(f_{k+1}\right)=A_{k+1, k+1}(g) f_{k+1}$. Since there are no nonzero 
stationary points, $A_{k+1, k+1}(g) \neq 1$, for some $g \in G$. This holds for $k=0, \ldots, n$ -1 .

3.3. TheOREM. Let $G$ be a connected nilpotent group. If $E^{G}=0$ then there exists $g \in G$ with $I-g$ invertible on $E$.

Proof. By the Lie-Kolchin theorem (see e.g. Humphreys [8]), because $G$ is connected and solvable, its natural action on $\mathrm{C} \otimes E$ is represented by upper-triangular matrices $\left[A_{i j}(g)\right]$. Let $\Gamma \subset \mathrm{GL}(\mathrm{C} \otimes E)$ be the closure of the set of all these matrices. Then $\Gamma$ is a connected Lie group, nontrivial because $G$ does not act trivially. Let $\Gamma_{i}=\left\{A \in \Gamma: A_{i i}=1\right\}$. Each $\Gamma_{i}$ is a closed subgroup, and $\Gamma_{i} \neq \Gamma$ by Lemma 3.2. Moreover $\operatorname{dim} \Gamma_{i}<\operatorname{dim} \Gamma$ because $\Gamma$ is connected. It follows that $\Gamma-\cup \Gamma_{i}$ is a nonempty open subset of $\Gamma$; hence it contains some matrix $\left[A_{i j}(g)\right.$ ], $g \in G$. None of the diagonal entries $A_{i i}(g)$ is 1 ; it follows that 1 is not an eigenvalue of $\left[A_{i j}(g)\right]$. This means that $I-g$ is invertible on $E$.

Proof of Theorem C. (a) $\Rightarrow$ (b). Stability of the origin implies $H^{1}(G, E)=0$ by Theorem 1.1. Since $G / G^{\prime}$ is a noncompact abelian Lie group, $\operatorname{Hom}(G, \mathbf{R}) \neq 0$. Therefore $E^{G}=0$ by Theorem B.

(b) $\Rightarrow$ (c). This follows from Theorem 3.3.

(c) $\Rightarrow$ (b). Obvious.

(b) $\Rightarrow(a)$. This is a special case of the following result of Dennis Stowe [10].

TheOREM OF D. STOWE. Let $p \in M$ be a stationary point of a smooth action of $a$ compactly generated nilpotent group on a manifold $M$. If the linearized action on the tangent space at $p$ has the origin as its only stationary point, then $p$ is stable.

Proof of Theorem D. If the origin is stable then by Theorem $\mathrm{B}, E^{G}=0$. The converse follows from Stowe's theorem.

REMARK. Theorem D applies when $G$ is discrete, finitely-generated, nilpotent and torsion-free. To see that $\operatorname{Hom}(G, \mathbf{R}) \neq 0$ in this case we proceed by induction on the nilpotence of $G$. The case where $G$ is abelian is trivial. Let $Z \subset G$ be the center. It suffices to prove that $G / Z$ is torsion-free, for the induction hypothesis will then prove that $\operatorname{Hom}(G / Z, \mathbf{R}) \neq 0$. Suppose $x^{n} \in Z$ for some $n>0$. Then for all $y$ in $G$ we have $\left(y x y^{-1}\right)^{n}=x^{n}$. But $n$th roots are unique in torsion-free nilpotent groups (Kurosh [11]) so $y x y^{-1}=x$. Therefore $x \in Z$, proving that $G / Z$ is torsion-free.

\section{BIBLIOGRAPHY}

1. M. Atiyah and C. T. C. Wall, Cohomology of groups, Algebraic Number Theory, edited by Cassels and Frohlich, Thompson, Washington, D. C., 1967, pp. 94-115.

2. W. Dwyer, Vanishing homology over nilpotent groups, Proc. Amer. Math. Soc. 49 (1975), 8-12.

3. D. Fried, W. Goldman and M. Hirsch, Affine manifolds (in preparation).

4. M. Hirsch, Differential topology, Graduate Texts in Math., No. 33, Springer-Verlag, Berlin and New York, 1976.

5. __ Flat manifolds and the cohomology of groups, Proc. Sympos. Geometric Topology in honor of Raymond Wilder, Springer-Verlag, New York, 1978. 
6. On stability of stationary points of transformation groups, Bifurcation Theory and Applications in Scientific Disciplines, edited by O. Gurel and O. Rössler, Ann. New York Acad. Sci., vol. 316, 1979.

7. __ Stability of stationary points of group actions, Ann. of Math. (2) 109 (1979), 537-544.

8. J. Humphreys, Linear algebraic groups, Graduate Texts in Math., No. 21, Springer-Verlag, Berlin and New York, 1975.

9. J. Stasheff, Continuous cohomology of groups and classifying spaces, Bull. Amer. Math. Soc. 84 (1978), 513-530.

10. D. Stowe, Actions of nilpotent groups, Univ. of California, Berkeley, 1977 (preprint).

11. A. Kurosh, Theory of groups, Vol. 2, 2nd ed., Chelsea, New York, 1960.

Department of Mathematics, University of California, Berkrley, California 94720 